\title{
Purification Efficiency on the Granulometric Composition
}

\author{
${ }^{1}$ IInar A. Nasyrov, ${ }^{2}$ Dinar D. Fazullin, ${ }^{3}$ Gennady V. Mavrin, ${ }^{4}$ Ildar G. Shaikhiev \\ ${ }^{1-3}$ Kazan Federal University \\ ${ }^{4}$ Kazan National Research Technical University \\ Email:chem_aleb@mail.ru
}

Received: $20^{\text {th }}$ August 2019, Accepted: $3^{\text {th }}$ September 2019, Published: $3^{\text {st }}$ October 2019

\begin{abstract}
In this work, studies were carried out on sorption treatment in dynamic conditions of the model solution of copper ions (II) sorbing material obtained by the treatment of sediment biological wastewater treatment with low-temperature pyrolysis. Research conducted for fractions with particle sizes less than $0.5 \mathrm{~mm}$ and $0.5-1 \mathrm{~mm}, 1-2 \mathrm{~mm}, 2-3 \mathrm{~mm}, 3 \mathrm{~mm}$ or more.

Dynamic sorbing of the model solution was carried out through a product of pyrolysis with a certain size of particles filled in a column with a diameter of $10 \mathrm{~mm}$, the mass of sorbent was 2 grams, the height of filling the column $10-12 \mathrm{~cm}$. The speed at which the model solution is filtered through the column depends on the size of the particles of the sorption material. As the particle size increases, the filtration rate increases, but the efficiency of copper ions removal decreases. Degree of sorption of copper ion solution with initial concentration of $1028 \mathrm{mg} / \mathrm{dm}^{\wedge} 3$ amounted to $94.6 \%$ for fraction of pyrolysis product with particle size $1-2 \mathrm{~mm}, 91.6 \%$ - less than $0.5 \mathrm{~mm}, 92.1 \%-0.5-1 \mathrm{~mm}, 91.2 \%-$ more than $2 \mathrm{~mm}$ respectively. To compare the degree of sorption for BAU-grade coal amounted to $93.1 \%$. Speed of filtering for BAUgrade coal amounted to $10 \mathrm{~mL} / \mathrm{min}$.

The size of the particles of the dispersal phase of the original model solution and filter after sorption was determined by dynamic light scattering (DLS) using the NanoBrook Omni analyzer. According to the results of the study, the model solution is a polydisperse system with particle sizes from 145 to $224 \mathrm{~nm}$ and 6450 to $8640 \mathrm{~nm}$. The size of the particles of the dispersal phase of the filter after passing through the sorbing material with the size of particles $1-2 \mathrm{~mm}$ is in the area from 122 to $177 \mathrm{~nm}$ and from 1280 to $2380 \mathrm{~nm}$. The peaks of particle size in the filter after passing through the sorbent (a fraction of 1-2 mm) are shifted downwards, indicating that smaller particles remain in the filter after cleaning. This is confirmed by the increase in copper ions.
\end{abstract}

Keywords

Pyrolysis, Sludge, Sorbent, Model Solution, Filtration Rate, Degree of Sorption.

\section{Introduction}

The current issue is the protection of the environment from pollution, the development of resource-saving and waste-free technologies. At the same time, the use of circulating water supply systems and the treatment of domestic and industrial wastewater is an important and demanding task.

As a result of household and industrial activity of the person waste in the form of sewage is formed. Wastewater, combining industrial, domestic and atmospheric, is a source of pollution of urban areas. [1]. All types of wastewater require pre-treatment before discharge into ponds, due to the content of various pollutants in them, the concentrations of which often exceed the maximum permissible.

For extraction of heavy metal ions (HMI) from the treated water, the reagent method is widely used, which is based on the introduction of chemicals into the aqueous medium that accelerates the processes of agglomeration and deposition of pollutants. Coagulation and flocculation facilitate the removal of formed colloids by concentrating them into flakes and floccules, followed by separation by settling, flotation and filtration [2]

Chemical deposition of impurities present in water involves the transfer to an insoluble state of one or more compounds. In this case, a soluble reagent is introduced into the purified water, which forms a precipitate, which is an insoluble compound with impurities to be removed. Simple neutralization of acidic effluents by alkaline reagents and deposition of HMI in the form of hydroxides is the most common method of water purification from this type of pollution [3].

Electrochemical methods, which include electrocoagulation, in their prevalence of use occupy the second place after the reagent [4]. During electrochemical purification in an aqueous medium, heavy metal impurities are reduced on the electrode or precipitated by interaction with products that are formed as a result of the cathode reaction.

Ion exchange is a physical and chemical exchange process between ions that are in the solution and are present on the surface of solid ionite. This method is widely used to purify contaminated water from HMI (copper, chromium, zinc, lead, nickel, cadmium, mercury, manganese, etc.) Deficiencies are the need for continuous ion exchanger regeneration, recycling solutions, formed after cleaning [5].

For the deep treatment of production wastewater from HMI, the method of sorbing is used, the efficiency of which can reach $80-95 \%$ and depends on the chemical nature of the adsorbent, its structure, capacity, specific surface, structure and properties of pollutants substances [5]. Dynamic adsorption is carried out by passing the purified water through a filter loaded with a layer of the sorbent. as adsorbents using Active carbons, carbon fiber materials, silica gels, synthetic sorbents. At present, affordable and relatively cheap sorbents derived from their waste are being used to clean it from 
HMI. The use of silt sediment after treatment with low-temperature pyrolysis as a sorption material helps to get rid of sile-accumulators and improve the quality of wastewater treatment [6]. Sorption properties of the product of pyrolysis are associated with the presence of carbon [7].

Each of the methods examined differs in its advantages and disadvantages, which requires more detailed study and improvement to use them efficiently and efficiently with the possibility of obtaining proper quality purified water, that can be dumped into natural ponds without harm ingests, or reused in a technological cycle.

The purpose of the paper is to define the dependence degree of purification of the model solution from the grain-size composition of sorption material obtained low-temperature pyrolysis of sludge biological wastewater treatment.

\section{Methods}

The distribution of solid product particles of silt precipitation pyrolysis by size was determined by the method of sith analysis and using a laser analyzer of the size of particles of the brand "Microsizer 201C" brand [8].

To assess the effectiveness of removing HMI used model solution with concentration of $1 \mathrm{~g} / \mathrm{l}$. Sorption cleaning model solution carried out on laboratory filtration installation (fig.1 [9]. Model aqueous solutions of a certain concentration were passed through columns filled with certain fractions of sorption material with a given flow rate. The height of the layer of sorption material $100 \mathrm{~mm}$ in the column with inner diameter $10 \mathrm{~mm}$, length $150 \mathrm{~mm}$, weight filler was 2 grams. Research conducted for fractions with particle sizes less than $0.5 \mathrm{~mm}$ and $0.5-1 \mathrm{~mm}, 1-2 \mathrm{~mm}, 2-3 \mathrm{~mm}, 3 \mathrm{~mm}$ or more. The size of the particles of the dispersal phase of the original model solution and filter after sorption was determined by dynamic light scattering (DLS) using the NanoBrook Omni analyzer [9].

The main indicators of the dynamic sorption cleaning model solution considered performance and degree of sorption.

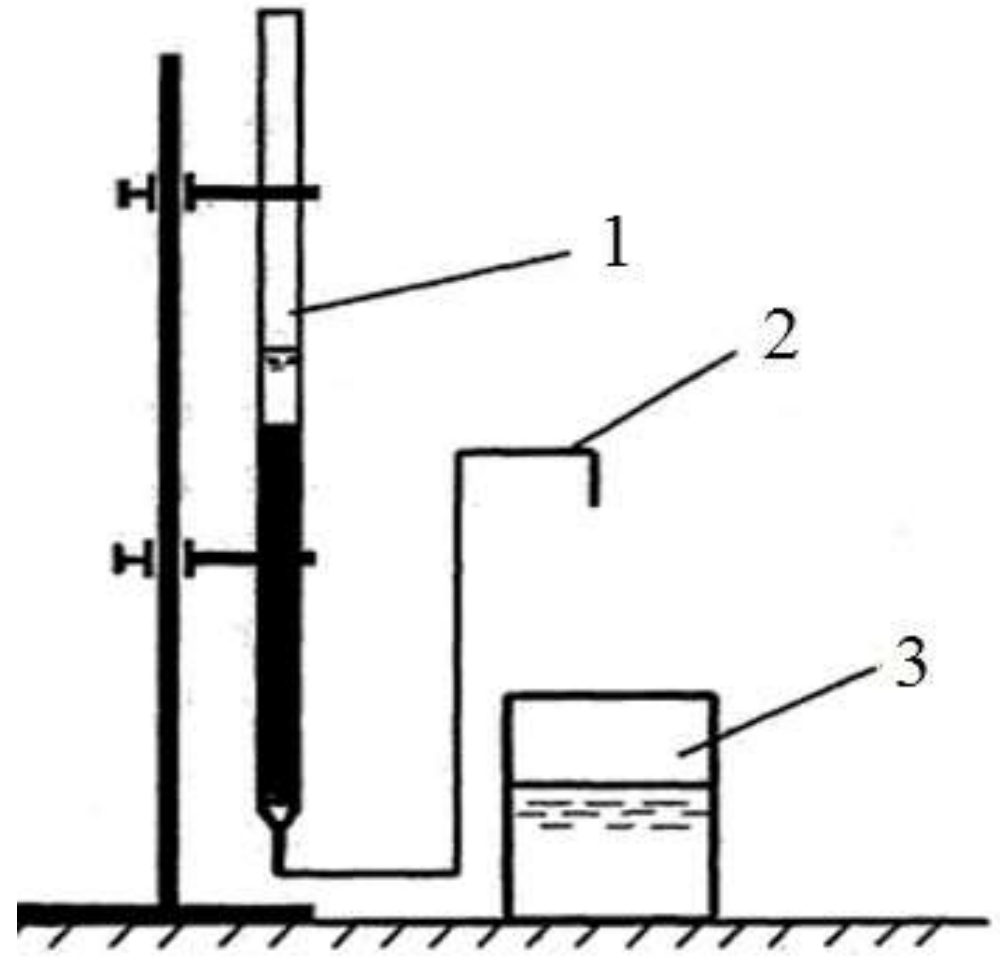

Figure 1: Scheme of Filtering Installation

[1:Sorption Column with Adsorbent; 2: Withdrawal of a Filtrate; 3: Container]

\section{Results and Discussion}

This work investigated the sorption material produced by the low-temperature pyrolysis of sludge biological wastewater treatment. The product of pyrolysis is a black powder with grayish shade without extraneous inclusions. The appearance of the product of pyrolysis of sludge is shown in Figure 2. 


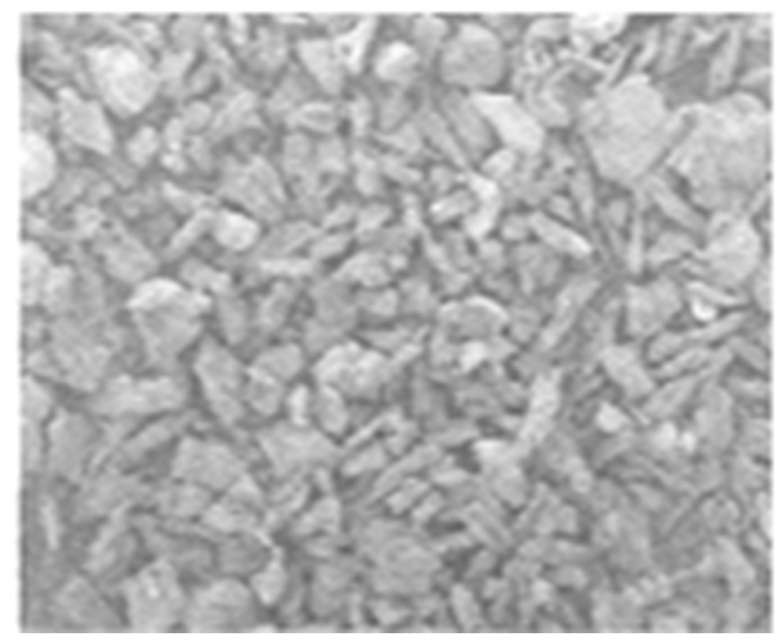

Figure 2: Solid Product of the Processing of Sludge of Sewage Sewer

The results of a study of the microstructure using scanning electron microscope Jeol JSM-brand 6390 LA "indicates that a product of pyrolysis of sludge has a porous structure (fig. 3). The porous structure allows for predicting sorption properties.

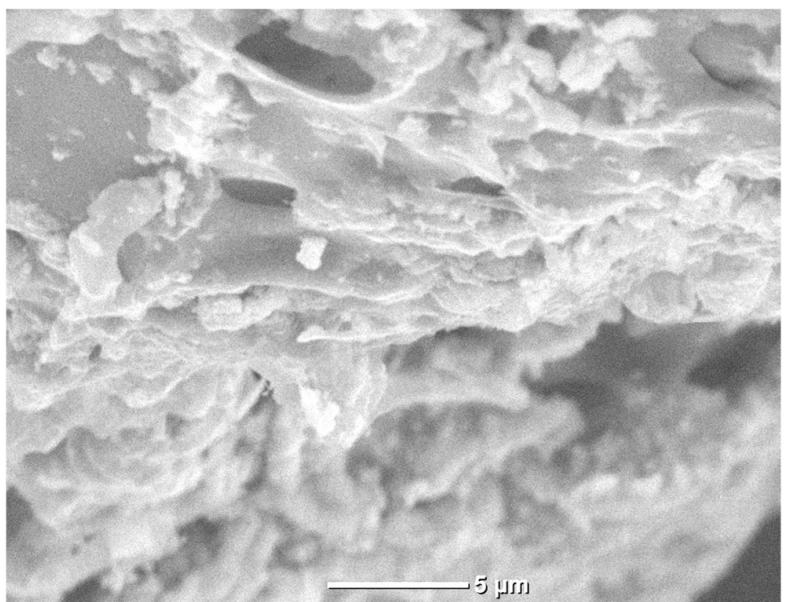

Figure 3: Structure of the Product of the Development of Sludge

The distribution of solid product particles of silt precipitation pyrolysis by size was determined by the method of sith analysis and using a laser analyzer of the size of particles of the brand "Microsizer 201C" brand [8].

\begin{tabular}{|c|c|}
\hline Particle Size & content, $\%$ \\
\hline$>5$ & 22.3 \\
\hline $3-5$ & 20.9 \\
\hline $1-3$ & 29.3 \\
\hline $0.5-1$ & 9.9 \\
\hline $0.1-0.5$ & 12.3 \\
\hline $0.05-0.1$ & 1.7 \\
\hline $0.01-0.05$ & 1.8 \\
\hline$<\mathbf{0 . 0 5}$ & $\mathbf{1 . 8}$ \\
\hline
\end{tabular}

Table 1: Particle Size Distribution (\%)

Conducted by granulometric analysis showed that solid sludge pyrolysis product contains mostly particles with sizes from 0.1 to over $5 \mathrm{~mm}(82.4 \%)$. The presence in the sample of large particles with sizes of more than $1 \mathrm{~mm}$ is due to the content of silt particles that are not decomposed during pyrolysis.

For further study, fractions of less than $0.5 \mathrm{~mm}, 0.5-1 \mathrm{~mm}, 1-2 \mathrm{~mm}, 2-3 \mathrm{~mm}, 3 \mathrm{~mm}$ and more were selected, which prevail in the product of pyrolysis of silt precipitation.

Initially, specific performance was studied, which was defined as the ratio of the amount of filter generated and the processing time in terms of $\mathrm{ml} / \mathrm{min}$.

Figure 4 shows the dependence of filtration speed through the column on the size of particles of the product of pyrolysis of silt precipitation. 


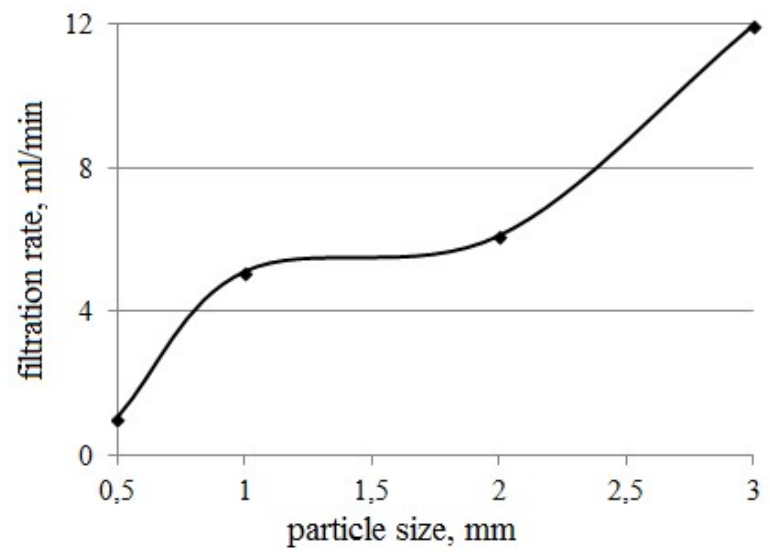

Figure : A Plot of Dependence of Filtration Rate on the Size of Particles of Pyrolysis Product of Sludge

The filtration rate of the model solution through the column filled with sorption material is minimal at a fraction of less than $0.5 \mathrm{~mm}$. When the particle size increases to $1 \mathrm{~mm}$, the filtration rate increases dramatically 5-6 times. The speed of the model filtering solution through pyrolysis product particles in the size range of 1 to $3 \mathrm{~mm}$ is amplified in 2 times. For comparison, studies were conducted on the filtration rate for coal brand BAU, which was $10 \mathrm{ml} / \mathrm{min}$.

To study the process of copper ions (II) in dynamic conditions, they used the model solution $\mathrm{Cu}^{2+}$ with a concentration of $1 \mathrm{~g} / 1\left(1028 \mathrm{mg} / \mathrm{dm}^{\wedge} 3\right)$. The initial and final concentrations of copper ions were determined by atomic-absorption spectroscopy on the KVANT-Z.ETA spectrometer.

The results determine the sorption properties of the sludge pyrolysis product concerning the copper ions are shown in table 2 .

\begin{tabular}{|l|c|}
\hline \multicolumn{1}{|c|}{ Sample } & concentration, $\mathrm{mg} / \mathrm{dm}^{\wedge} 3$ \\
\hline stock solution & 1028 \\
\hline the filtrate from product development, particle size $<0.5 \mathrm{~mm}$ & 86.6 \\
\hline the filtrate from product development, particle size $0.5-1 \mathrm{~mm}$ & 81.3 \\
\hline the filtrate from product development, particle size $1-2 \mathrm{~mm}$ & 55.6 \\
\hline the filtrate from pyrolysis product, particle size $>2 \mathrm{~mm}$ & 90.5 \\
\hline activated carbon (BAU) & 71.2 \\
\hline
\end{tabular}

\section{Table 2: Mass Content of Heavy Metal Ions}

For comparison, Table 2 provides studies of bau coal sorption, which is a common sorption material.

Sorption (R, \%) calculated by the formula (1):

$R=\frac{C_{0}-C_{1}}{C_{0}} * 100$

where $\mathrm{C}_{0}$ - the initial concentration of the ITM, $\mathrm{mg} / \mathrm{dm}^{\wedge} 3$;

$\mathrm{C}_{1}$ - concentration of HMI after sorption, $\mathrm{mg} / \mathrm{dm}^{\wedge} 3$.

The results of the sorption calculation are presented in Figure 4.

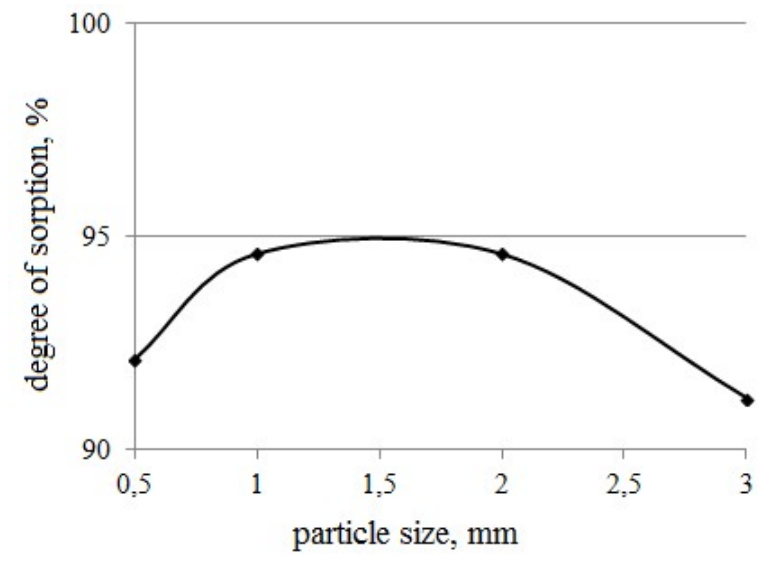

Figure 4: A Plot of Dependence of Filtration Rate on the Size of Particles of Pyrolysis Product of Sludge 
The cleaning efficiency of the model solution of copper ions was $91-95 \%$, depending on the size of the particles of a solid product of pyrolysis of sludge, and the degree of sorption of coal of grade BAU-93.1\%. Increasing the size of the particles leads to a decrease in the efficiency of purification of copper ions.

Figure 5 shows a graph of the distribution of the size of the particles of the dispersive phase of the $\mathrm{CuSO}_{4}$ model solution. According to Figure 5, the original model solution is a polydisperse system with particle sizes from 145 to $224 \mathrm{~nm}$, and 6450 to $8640 \mathrm{~nm}$.

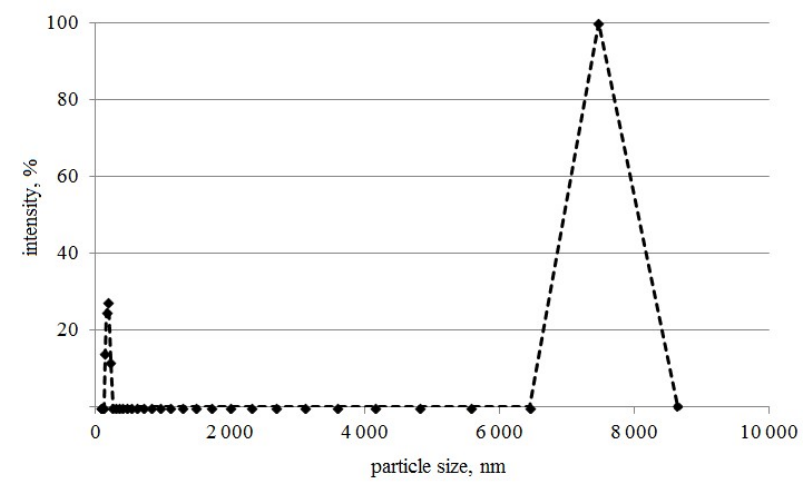

Figure 5: A Plot of the Particle Size Distribution of the Dispersed Phase of the Model Solution

Figure 6 charts the distribution of the size of the dispersal phase particles after filtering through a layer of sorption material for different fractions (less than $0.5 \mathrm{~mm}, 0.5-1 \mathrm{~mm}, 1-2 \mathrm{~mm}, 2 \mathrm{~mm}$ or more).

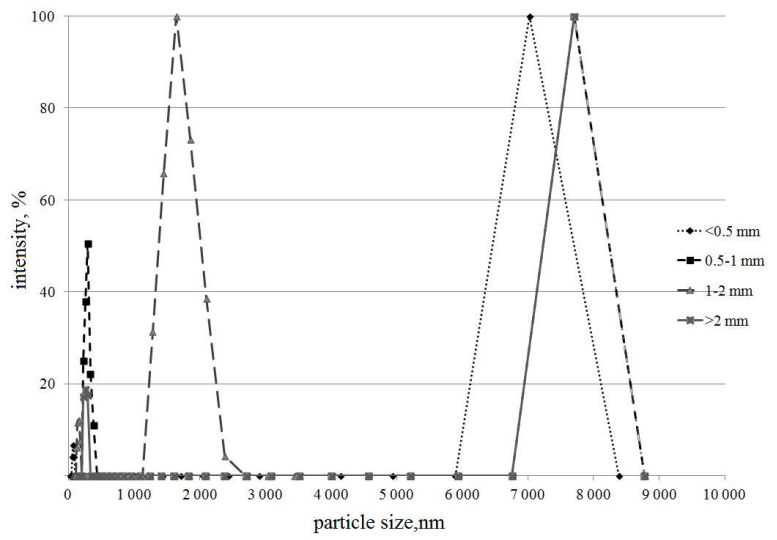

Figure 6: A Plot of the Particle Size Distribution of the Disperse Phase of the Model Solution after Filtration through the Pyrolysis Product of Sludge

It is shown that the size of disperse phase particles filtrate after passing the model solution through sorption material product of pyrolysis of sludge with particle sizes less than $0.5 \mathrm{~mm}$ is located in an area from 60 up to $85.4 \mathrm{~nm}$ and from 5890 to $8380 \mathrm{~nm}$. The size of the particles of the dispersal phase of the filter after passing through the sorbing material with the size of particles $0.5-1 \mathrm{~mm}$ is in the area from 226 to $382 \mathrm{~nm}$ and from 6760 to $8780 \mathrm{~nm}$, with particle size more than $2 \mathrm{~mm}$ - from 226 to $294 \mathrm{~nm}$ and from 6760 to $8780 \mathrm{~nm}$ and the size of the particles of the dispersal phase of the filter after passing through the sorption material with the size of particles 1-2 mm - from 122 to $177 \mathrm{~nm}$ and from 1280 to 2380 $\mathrm{nm}$, respectively. The peaks of particle size in the filter after passing through the sorbent (a fraction of 1-2 mm) are shifted downwards, indicating that smaller particles remain in the filter after cleaning. This fact confirms the increase in copper ions $(94.6 \%)$. 


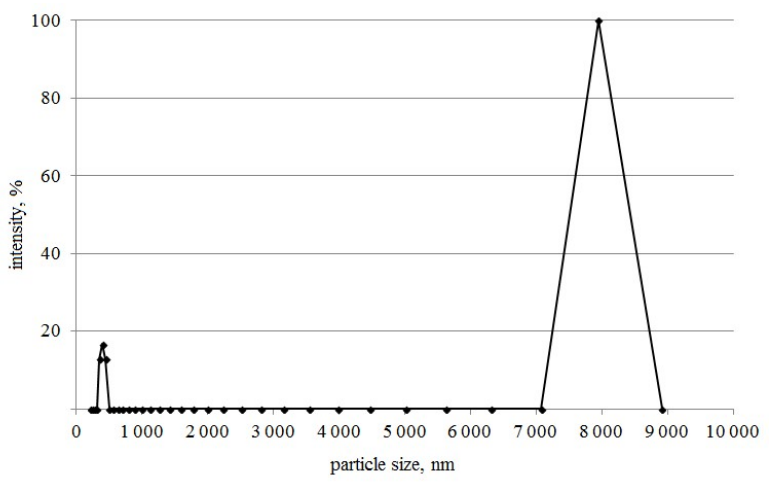

Figure 7: A Plot of the Particle Size Distribution of the Disperse Phase of the Model Solution after Filtration through BAU

\section{Summary}

According to the results of the study, the model solution is a polydisperse system with particle sizes from 145 to $224 \mathrm{~nm}$ and 6450 to $8640 \mathrm{~nm}$. The size of the particles of the dispersal phase of the filter after passing through the sorbing material with the size of particles $1-2 \mathrm{~mm}$ is in the area from 122 to $177 \mathrm{~nm}$ and from 1280 to $2380 \mathrm{~nm}$. The peaks of particle size in the filter after passing through the sorbent (a fraction of 1-2 mm) are shifted downwards, indicating that smaller particles remain in the filter after cleaning.

The speed at which the model solution is filtered through the column depends on the size of the particles of the sorption material. As the particle size increases, the filtration rate increases, but the efficiency of copper ions removal decreases. For a fraction of the 1-2 filtering, speed is $6 \mathrm{ml} / \mathrm{min}$. For comparison, studies were conducted on the filtration rate for coal brand BAU, which was $10 \mathrm{ml} / \mathrm{min}$.

The cleaning efficiency of the model solution of copper ions was $91-95 \%$, depending on the size of the particles of a solid product of pyrolysis of sludge, and the degree of sorption of coal of grade BAU-93.1\%. Increasing the size of the particles leads to a decrease in the efficiency of purification of copper ions.

Particle size is optimal sludge pyrolysis product in the range of 1 to $2 \mathrm{~mm}$, where there is a high degree of removal of copper ions (94.6\%), and compared with the BAU acceptable speed filtering $(6 \mathrm{ml} / \mathrm{min})$.

\section{Conclusions}

The copper ion removal efficiency of the solution depends on the particle size of the sorption material obtained sludge pyrolysis. Increasing the size of the particles leads to a decrease in the efficiency of purification of copper ions. Removal efficiency also depends on the size of the particles of the dispersed phase modeling solution.

The sorption process flows through the layer of sorption material with particle sizes 1-2 mm during filtering through the column under dynamic conditions, we recommend that you use for the preliminary purification of wastewater.

\section{Acknowledgments}

The work is performed according to the Russian Government Program of Competitive Growth of Kazan Federal University.

\section{References}

[1] I.A. Nasyrov Research of sludge pyrolysis properties of the product//proceedings of the 7th International Congress "Pure water. Kazan ". -Kazan: LLC "new knowledge". -2016. -pp. 247-250

[2] V.V. Semenov Improving the effectiveness of the reagent production of oily wastewater treatment/V.V. Semenov, S.N. Rodionov, A.F. Naslednikova // Water and sanitary equipment. -2017. -No. 8. -pp. 34-39.

[3] Yunsi Aziz. Improving the reliability and environmental safety of wastewater treatment systems of radio-electronic industry enterprises: the dissertation for the degree of candidate of technical sciences. Moscow State University of Technology, Moscow, 2017.

[4] E.V. Alekina Analysis of wastewater treatment methods used in engineering enterprises / E.V. Alekina, I.A. Sumarchenkova // Water treatment, water treatment, water supply. - 2018 .- No. 1 (97). - pp. 56-61.

[5] A.A. Nekrasova. Investigation of the sorption properties of filtering materials / A. A. Nekrasova, D.M. Privalov, M.V. Dvadnenko, N.M. Privalova // Political Mathematical Network Electronic Scientific Journal of the Kuban State Agrarian University. - 2017. - No. 126. - pp. 266-275.

[6] Sorption properties of carbon waste pyrolysis product for biological wastewater treatment. Ilnar A. Nasyrov, Stanislav V. Dvoryak, and Ildar G. Shaikhiev // Acta Technica. - 2016.-№61 (4B / 2016), pp. 307-314.

[7] Petroleum containing wastewater products purification by carbon-containing wastes pyrolysis products. Ilnar A. Nasyrov, Aigul I. Ahmadieva, Dinar D. Fazullin, Gennady V. Mavrin, and Mikhail P. Sokolov // The Turkish Online Journal of Design, Art and Communication TOJDAC. - 2017.-№ 7 SE., pp. 1713-1728. 
[8] Sorption properties of pyrolysis products of sludge, wood waste and rubber waste for heavy metal ions. Ilnar A. Nasyrov, Gennady V. Mavrin, Aliya R. Ahmetshina, and Aigul I. Ahmadieva // Journal of Fundamental and Applied Sciences. - 2017.-№ 9 (1S), pp. 1615-1625.

[9] Removal of oil products and phenol from wastewater by composite sorbents under dynamic conditions / D.D. Fazullin, G.V. Mavrin, R.G. Melkolvan. // Chemistry and Technology of Fuels and Oils. - 2014. - N 1. -pp. 8894.

[10] Particle Size and Zeta Potential Changes in the Disperse Phase of Water-Emulsified Waste Waters in Different Treatment Stages. D.D. Fazullin, I.G. Shaikhiev, G.V. Mavrin. / Chemistry and Technology of Fuels and Oils.2015. -Vol. 51. No. 5. -pp. 501-504. 